\title{
Using an Integrated Artificial Neural Networks Model for Predicting Global Radiation: The Case Study of Iran
}

\author{
A. Azadeh, A. Maghsoudi and S. Sohrab khani \\ Research Institute of Energy Management and Planning, Department of Industrial Engineering \\ and Department of Engineering Optimization Research \\ Faculty of Engineering, University of Tehran, Iran \\ aazadeh@ut.ac.ir, maghsoodi@engmail.ut.ac.ir
}

\begin{abstract}
In this article we have used artificial neural networks for predicting solar global radiation by using climatological variables in the locations that no direct measurement equipment is available. Numbers of climatological and meteorological parameters were considered for this purpose and monthly data provided for 6 years (19952000) in 6 nominal cities in Iran. Separate model for each city is considered and the quantity of solar global radiation in each city is calculated. The average value of acquired results of these models has shown high accuracy about $94 \%$ and the mean absolute percentage error (MAPE) of the models was $6.7 \%$. The results of these models have proved the capability of ANN models for predicting solar radiation in the locations that there is no any measurement equipment. The results of this study have shown a better accuracy than other conventional prediction models that have been used up to now.
\end{abstract}

\section{Keywords}

Global Radiation, Solar Radiation, Artificial Neural Networks, Climatological Parameters, Forecasting.

\section{1- Introduction}

It has been recently realized that renewable energy sources and systems such as solar energy can have a beneficial impact with regard to essential technical, environmental, and political vision. Solar energy is the most ancient source, and it is the root material for almost all fossil and renewable types. Solar energy is freely available and could be easily harnessed to reduce our reliance on hydrocarbon-based energy by both, passive and active designs. Precise solar-radiation estimation tools are critical in the design of solar systems.

Using solar energy necessitates an exact estimation of solar energy in proposed locations. This matter usually is possible via solar measurement equipments but these devices are not available in some of remote or rural locations that specially have potential of solar installation. Using prediction tools as solar models is one of the best methods to have a good estimation of solar potential.
Using artificial neural network as an estimation tool has proved its efficiency in predicting different parameters via other parameters that their relationship is not specified. Climatological and meteorological parameters are important parameters in indicating the amount of solar radiation in a selected region. So applying artificial neural networks can be valuable in determining the effects of meteorological parameters and finally prediction of solar radiation.

There have been several articles that have used artificial neural networks for predicting solar radiation. The first works in this filed were presented by Al-Alawi \& Al-Hinai, 1998, Mohandes et al., 1998 and Lopez et al., 2001. In The article of Al-Alawi \& Al-Hinai, 8 different parameters as the inputs of ANN model is used for predicting solar energy in Oman. Their model has shown good accuracy about $93 \%$. A. Sozen et al. applied ANN model for forecasting solar radiation in Turkey. In this article 6 different input parameters were used and finally an accuracy about $93 \%$ was acquired (A. Sozen, 2005). In the work of F.S. Tymvios et al. 4 parameters as the inputs of the model were totally used. The ANN model with different forms of these input parameters was run and finally the acquired results of ANN model were compared with the results of conventional angstrom model (F.S. Tymvios, 2005).

In this study more climatological indicators have been used for predicting solar radiation that it can provide better results in outputs of the model. In this article we have used 9 different parameters as inputs of ANN model (location, month, mean of maximum temperature, mean of minimum temperature, mean relative humidity, mean vapor pressure, total precipitation, mean of wind speed and mean duration of sunshine). These parameters are the most cited climatological parameters in the previous studies. Data for these parameters are provided from national meteorological organization of Iran. For this purpose monthly mean of daily meteorological parameters were used. These data were collected for 6 different cities in Iran that are located in the different climatological regions. Monthly mean of daily solar global radiation was chosen as the output of ANN model. 


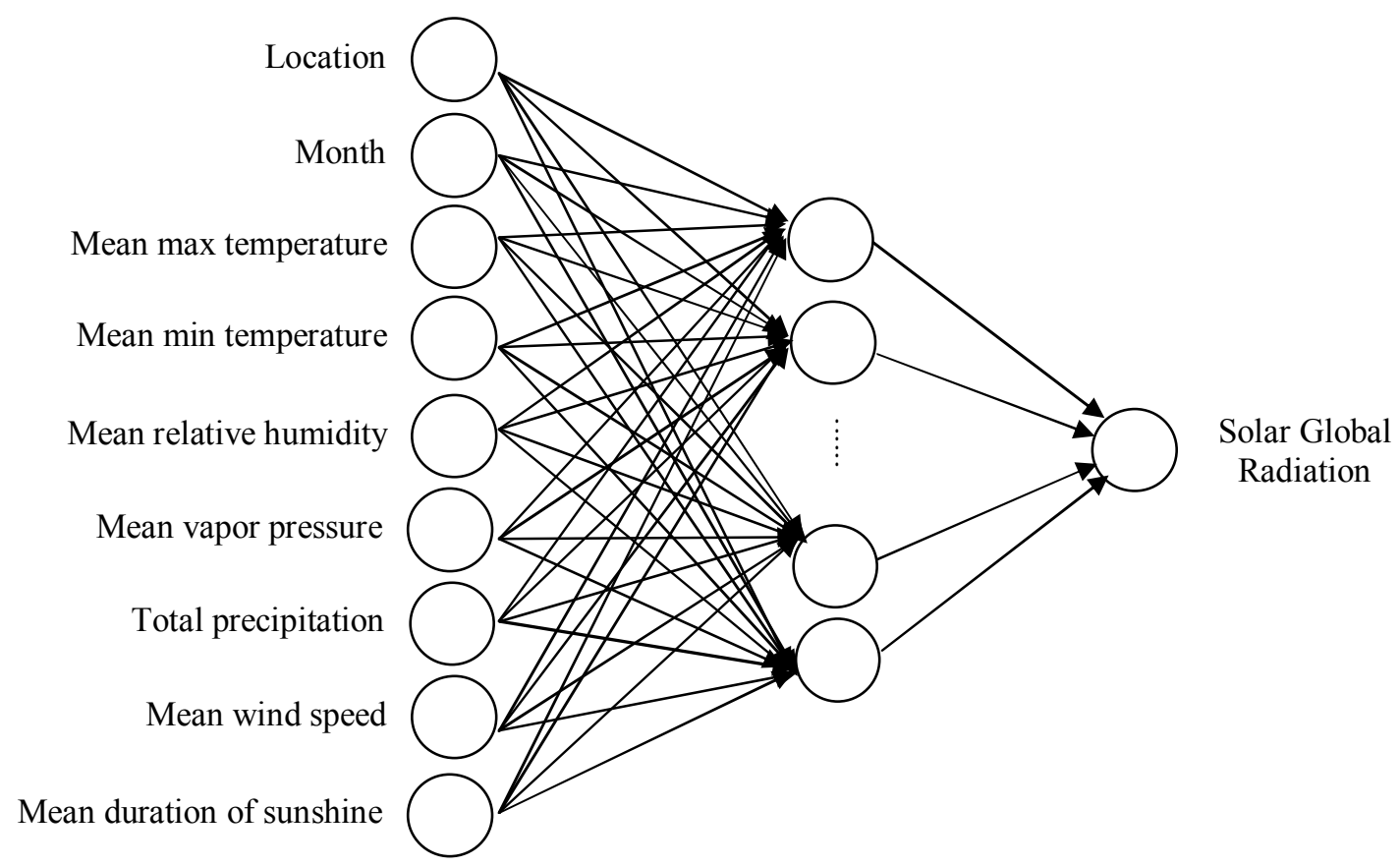

Fig. 1. ANN architecture for the global radiation prediction model

\section{2- Artificial Neural Networks}

In general, ANNs are simply mathematical techniques designed to accomplish a variety of tasks. The research in the field has a history of many decades, but after a diminishing interest in the 1970 's, a massive growth started in the early 1980's. Today, Neural Networks can be configured in various arrangements to perform a range of tasks including pattern recognition, data mining, classification, forecasting and process modeling. ANNs are composed of attributes that lead to perfect solutions in applications where we need to learn a linear or nonlinear mapping. Some of these attributes are: learning ability, generalization, parallel processing and error endurance. These attributes would cause the ANNs solve complex problem methods precisely and flexibly.

ANNs consists of an inter-connection of a number of neurons. There are many varieties of connections under study, however here we will discuss only one type of network which is called the Multi Layer Perceptron (MLP). In this network the data flows forward to the output continuously without any feedback. Figure 2 shows a typical three-layer feed forward model used for forecasting purposes. The input nodes are the previous lagged observations while the output provides the forecast for the future value. Hidden nodes with appropriate nonlinear transfer functions are used to process the information received by the input nodes. The model can be written as:

$$
y_{t}=\alpha_{0}+\sum_{j=1}^{n} \alpha_{j} f\left(\sum_{i=1}^{m} \beta_{i j} y_{t-i}+\beta_{0 j}\right)+\varepsilon_{t}
$$

Where $\mathrm{m}$ is the number of input nodes, $\mathrm{n}$ is the number of hidden nodes, $\mathrm{f}$ is a sigmoid transfer function such as the logistic: $f(x)=\frac{1}{1+\exp (-x)} \cdot\{\alpha \mathrm{j}, \mathrm{j}=0,1, \ldots, \mathrm{n}\} \quad$ is a vector of weights from the hidden to output nodes and $\left\{\beta_{i j}, i=1,2, \ldots, \mathrm{m} ; j=0,1, \ldots, \mathrm{n}\right\}$ are weights from the input to hidden nodes. $\alpha 0$ and $\beta$ oj are weights of arcs leading from the bias terms which have values always equal to 1. Note that Equation (1) indicates a linear transfer function is employed in the output node as desired for forecasting problems. The MLP's most popular learning rule is the error back propagation algorithm. Back Propagation learning is a kind of supervised learning introduced by Werbos (1974) and later developed by Rumelhart \& McClelland (1986). At the beginning of the learning stage all weights in the network are initialized to small random values. The algorithm uses a learning set, which consists of input desired output pattern pairs. Each input - output pair is obtained by the offline processing of historical data. These pairs are used to adjust the weights in the network to minimize the Sum Squared Error (SSE) which measures the difference between the real and the desired values over all output neurons and all learning patterns. After computing SSE, the back propagation step computes the corrections to be applied to the weights. 


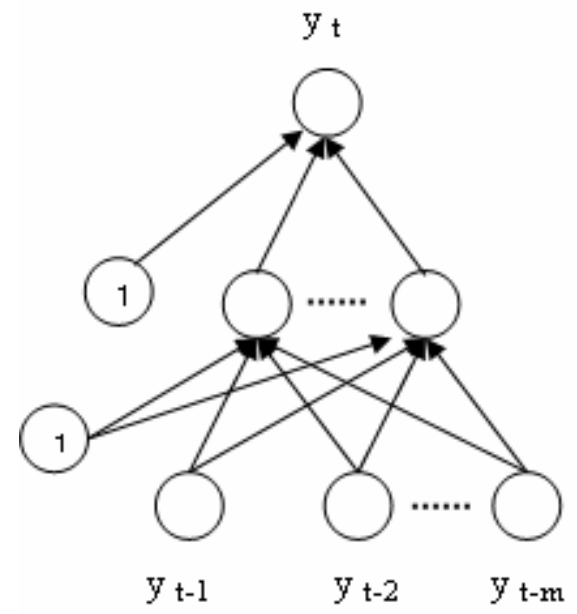

Fig. 2. A three layer MLP network.

The ANN models are researched in connection with many power system applications, short-term forecasting being one of the most typical areas. Most of the suggested models use MLP networks (J.H. Park et al., 1991, D.C. Park et al., 1991, T.M. Peng et al., 1992, T.M. Peng et al., 1993). The attraction of MLP has been explained by the ability of the network to learn complex relationships between input and output patterns, which would be difficult to model with conventional algorithmic methods.

There are three steps in solving an ANN problem which are 1) training, 2) generalization and 3) implementation. Training is a process that network learns to recognize present pattern from input data set. We present the network with training examples, which consist of a pattern of activities for the input units together with the desired pattern of activities for the output units. For this reason each ANN uses a set of training rules that define training method. Generalization or testing evaluates network ability in order to extract a feasible solution when the inputs are unknown to network and are not trained to network. We determine how closely the actual output of the network matches the desired output in new situations. In the learning process the values of interconnection weights are adjusted so that the network produces a better approximation of the desired output. ANNs learn by example. They cannot be programmed to perform a specific task. The examples must be selected carefully otherwise useful time is wasted or even worse the network might be functioning incorrectly. The disadvantage is that because the network finds out how to solve the problem by itself and its operation can be unpredictable. In this paper the effort is made to identify the best fitted network for the desired model according to the characteristics of the problem and ANN features.

\section{3- Data collection}

Six nominal cities in Iran were selected for using the proposed ANN model (Tehran, Esfahan, Kerman, Kermanshah, Zahedan, Bandar abbas). These cities have different climatological conditions and we can say that each of them is representative of one of climatological regions in Iran. Locations of these cities are presented in Figure 3. As mentioned earlier for predicting solar global radiation in the selected locations, we considered 9 different climatological parameters as the inputs of ANN model. Required data for using in the proposed ANN model was provided from national meteorological organization of Iran. These data were gathered for a period of six years from 1995 to 2000. For this purpose monthly mean of different input data such as maximum temperature, minimum temperature, relative humidity, vapour pressure, wind speed and duration of sunshine were calculated. For the input parameter precipitation total monthly value was calculated and used in the ANN model. Also number of month was used as one of the inputs. The single output parameter of the model is solar global radiation. For this parameter monthly mean of daily solar global radiation was used, too.

Totally, related data of 12 months for a period of 6 years (1995-2000) were gathered. We divided these set of data into two groups: the training subset and the testing subset. For the training subset related data of 65 months were considered and used for learning the model and for the testing subset related data of 7 months were used to test the capability of the model. 


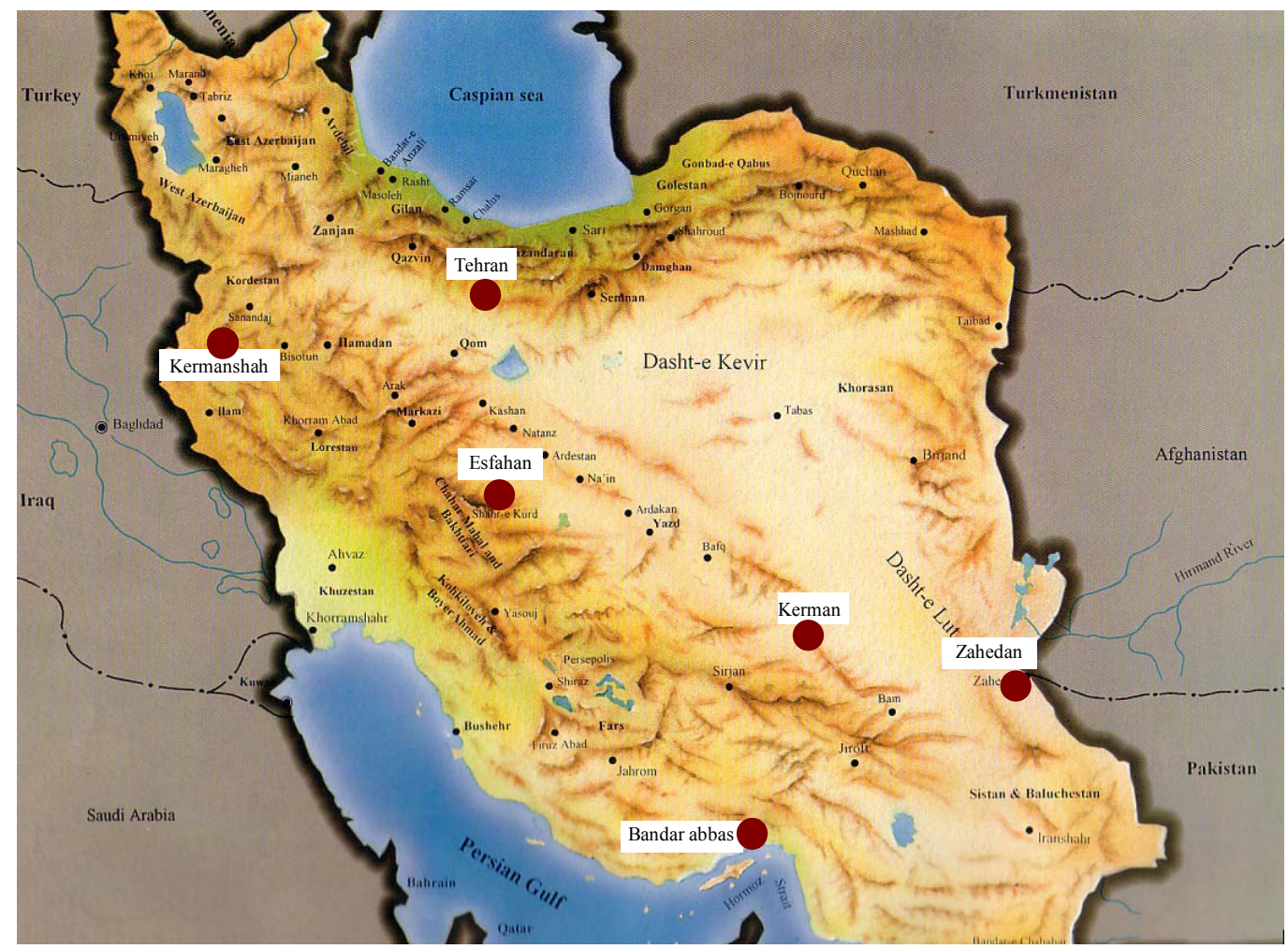

Fig. 3. The selected regions for using ANN model

\section{4- Running the ANN Model}

Finally to estimate monthly solar radiation, we introduce 7 variables for each month as the input variables of the model as follow:

$\&$ mean of maximum temperature

$\&$ mean of minimum temperature

$\&$ mean relative humidity

$\&$ mean vapor pressure

$\&$ total precipitation

$\& \quad$ mean of wind speed and

$\&$ mean duration of sunshine

And solar radiation is defined as the output variable.

Several MLP networks were generated and tested. The transfer function for the first layer was linear, for all hidden layers were sigmoid and for last one was linear. Back propagation algorithm was used to adjust the learning procedure and 7 rows of data selected to test the network. We used momentum and weight decay to improve the network. We also used another way of influencing the neural network called pruning. Pruning is the elimination of neuron connections which do not have a significant effect on the functionality of the network.

In order to decide which neuron connections have been eliminated from the network, the so-called "relevance" of a weight is examined. If the relevance after a series of learning epochs is below a certain threshold value, the connection is cut.

The results of the 6 best models and their errors for Bandar Abbas are shown in Table1. These results are derived from 7 rows of unlearned data. Mean absolute percentage error (MAPE) for each model is calculated from the following equation:

$$
e=\frac{1}{n} \sum_{i=1}^{n} \frac{\left|S R_{i(\text { estimated })}-S R_{i(\text { actual })}\right|}{S R_{i(\text { actual })}}
$$

Where SRi(estimated) is the estimated solar radiation for month $\mathrm{i}(\mathrm{i}=1,2, \ldots, 12)$ and SRi(actual) is the actual value of radiation. It can be seen that the 6 th model with 2 hidden layers and (7-6-2-1) neurons with pruning method has less error than other models. The graph of 6 th model is presented in Figure 4. As mentioned, these results were derived from 7 rows of unlearned data. 
TABLE I. - Different MLP results for Bandar Abbas

\begin{tabular}{|l|c|c|c|c|c|c|}
\hline MLP Model number & $\mathbf{1}$ & $\mathbf{2}$ & $\mathbf{3}$ & $\mathbf{4}$ & $\mathbf{5}$ & $\mathbf{6}$ \\
\hline $\begin{array}{l}\text { Number of neurons in } \\
\text { first hidden layer }\end{array}$ & 6 & 4 & 4 & 4 & 3 & 6 \\
\hline $\begin{array}{l}\text { Number of neurons in } \\
\text { second hidden layer }\end{array}$ & 2 & 2 & 0 & 0 & 0 & 2 \\
\hline Learning method & BP \& & BP \& & BP\& & BP \& & Back & BP\& \\
& $\& W e i g h t$ & momentum & Momentum & momentum & propagation(BP) & \&Weight \\
& decay & decay, & decay, & \&Weight & Momentum & decay, \\
& & pruning & pruning & Weight decay & pruning \\
\hline Relative error (MAPE) & 0.067 & 0.047 & 0.045 & 0.07 & 0.08 & 0.03 \\
\hline
\end{tabular}

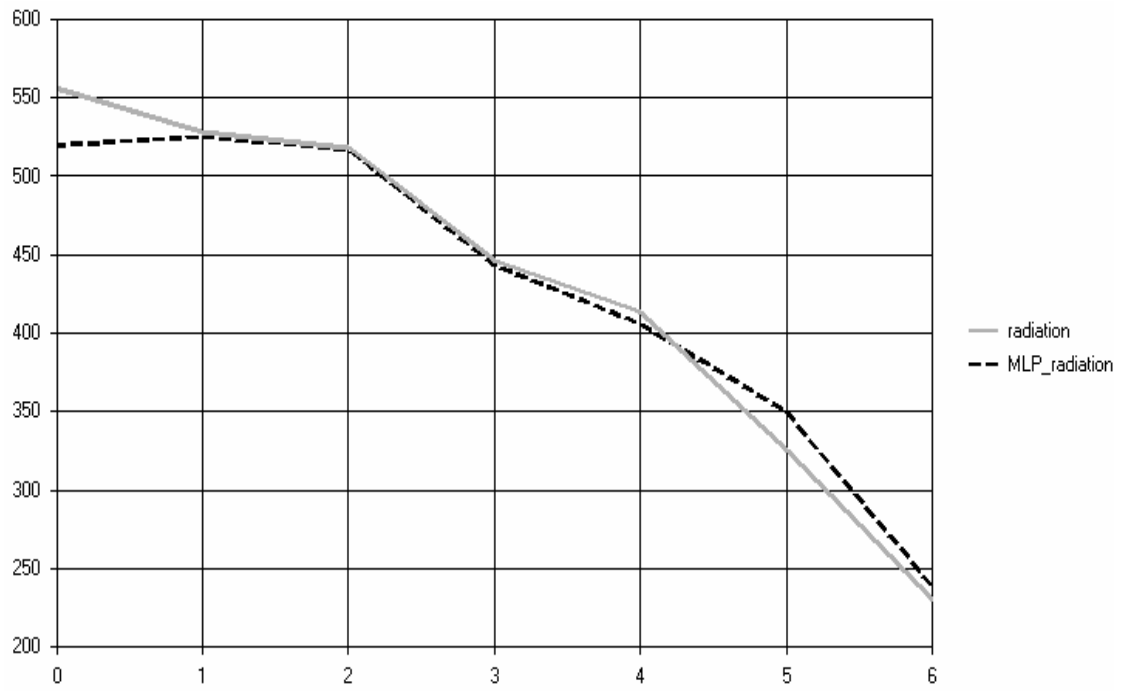

Fig. 4. Actual data versus the $6^{\text {th }}$ MLP results 
These efforts are also done for 5 other cities in different climatic regions and the results are shown in table 2. The mean actual values of 7 months versus MLP results for these cities are also depicted in Figure 5.

Furthermore to show the accuracy of the models two other parameters were considered. Errors during the learning stage namely as root mean squared error (RMS) and absolute fraction of variance (R2) are defined as bellow:

$$
\begin{aligned}
& R M S E=\sqrt{\frac{\sum_{i=1}^{n}\left(S R_{i(\text { estimated })}-S R_{i(\text { actual })}\right)^{2}}{n}} \\
& R^{2}=1-\left(\frac{\sum_{i=1}^{n}\left|S R_{i(\text { estimated })}-S R_{i(\text { (actual })}\right|^{2}}{\sum_{i=1}^{n}\left(S R_{i(\text { (actual })}\right)^{2}}\right)
\end{aligned}
$$

\begin{tabular}{|c|c|c|c|}
\hline City & $\begin{array}{l}\text { Best MLP } \\
\text { structure }\end{array}$ & MAPE & $\begin{array}{c}\text { Number of hidden } \\
\text { layer }\end{array}$ \\
\hline Bandar Abbas & $(7-6-2-1)$ & 0.03 & 2 \\
\hline Kerman & $(7-4-1)$ & 0.057 & 1 \\
\hline Kermanshah & $(7-4-1)$ & 0.067 & 1 \\
\hline Tehran & $(7-6-1)$ & 0.07 & 1 \\
\hline Esfehan & $(7-4-1)$ & 0.068 & 1 \\
\hline Zahedan & $(7-2-1)$ & 0.11 & 2 \\
\hline
\end{tabular}

TABLE II. - Different selected MLP networks for each city

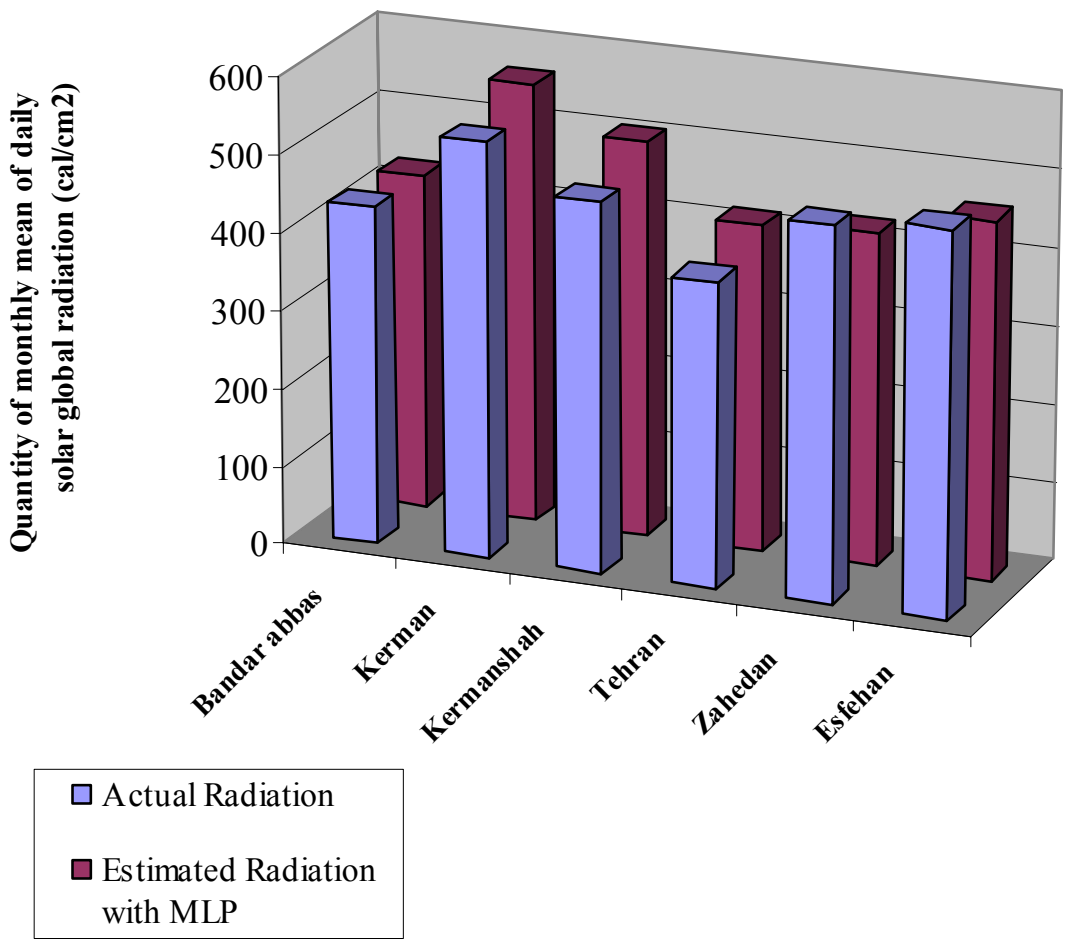

Fig. 5. Mean actual value of 7 months versus MLP results for 6 different climatic cities 
TABLE III. - The values of MAPE, RMSE and $\mathrm{R}^{2}$ for ANN results in each city

\begin{tabular}{|l|c|c|c|}
\hline \multicolumn{1}{|c|}{ City } & MAPE (\%) & RMSE (\%) & $\mathbf{R}^{\mathbf{2}}$ \\
\hline Bandar Abbas & 3 & 2.6 & 0.98 \\
\hline Kerman & 5.7 & 3 & 0.92 \\
\hline Kermanshah & 6.7 & 3.2 & 0.97 \\
\hline Tehran & 7 & 2.7 & 0.95 \\
\hline Esfehan & 6.8 & 3.3 & 0.97 \\
\hline Zahedan & 11 & 5.2 & 0.86 \\
\hline Average of the cities & 6.7 & 3.33 & 0.94 \\
\hline
\end{tabular}

Acquired results of RMSE and $\mathrm{R}^{2}$ validate the selected MLP structure for each model. As it seen the average of MAPE for all models is $6.7 \%$ and the average value of $R^{2}$ is 0.94 that shows the efficiency of ANN models for predicting solar radiation based on climatological parameters. Acquired results of this study show a considerable accuracy for predicting solar radiation in Iran. The best observed results of conventional methods such as angstrom model does exceed from the range of $10 \%$ in Iran that have a great difference with the results of ANN model. The results of this study emphasize the role of climatological parameters on the quantity of solar radiation

\section{5- Conclusion}

Several benefits of solar energy have made it as an important energy production tool. But using this energy requires having knowledge about radiation potential in different locations. Recently the use of artificial neural networks has developed widely as a prediction tool. In this article we have used an ANN model for predicting solar global radiation in Iran. For this model numbers of climatological and meteorological parameters were introduced and applied for predicting solar radiation. These parameters are the most conventional parameters that are available in many places. Application of this model shows better results rather than traditional prediction models and it could be used to evaluate the solar potential of a location. The use of these models in the remote locations that solar measurement devices are not available can be beneficial as an effective tool to select the most efficient locations for using solar energy.

\section{References}

A. Sozen, E. Arcaklıglu, M. Ozalp \& E. Galip Kanit, "Solar-energy potential in Turkey", Applied Energy 80 (2005) $367-381$

A. Sozen et al. Forecasting based on neural network approach of solar potential in Turkey Renewable Energy 30 (2005) 1075-1090
Al-Alawi,S.M., Al-Hinai,H.A., 1998. An ANN approach for predicting global radiation in locations with no direct measurement instrumentation. Renew. Energy $14,199-204$.

D.C. Park, M.A. El-Tarawa, R.J. Marks, L.E. Atlas, \& M. J. Dasburg, Electric load forecasting using an artificial neural network, IEEE Transactions on Power Systems, 6: No. 2, $442-449$ (1991).

D.E. Rumelhart, \& J.L. McClelland (Eds.), Parallel Distributed Processing: Explorations in the Microstructure of Cognition, Vol. 1, Foundations, MIT Press, Cambridge, MA, 1986.

F. Nielsen, "Neural Networks-algoritms and application", 2001 Niels Brock Bussines college.

F.S. Tymvios et al. Comparative study of Angstrom's and artificial neural networks methodologies in estimating global solar radiation. Solar Energy 78 (2005) 752 762

J.H. Park, Y.M. Park, \& K.Y. Lee, Composite modeling for adaptive short term load forecasting, IEEE Transactions on Power Systems, 6: No. 2, 450-457 (1991).

Lopez, G.,Rubio, M.A.,Martinez ,M.,Batlles, F.J.,2001. Estimation of hourly global photosynthetically active radiation using artificial neural network models. Agri. Forest Meteorol. 107,279 - 291.

Mohandes,M.,Rehman ,S.,Halawani, T.O.,1998. Estimation of global solar radiation using artificial neural networks. Renew. Energy 14,179 - 184.

P.I. Werbos, Beyond Regression: new tools for prediction and analysis in the behavior sciences. Ph.D. Thesis, Harvard University, Cambridge, MA, 1974.

T.M. Peng, N. Hubele, \& G.F. Karady, Advancement in the application of neural networks for short-term load forecasting, IEEE Transactions on Power Systems, 7: No. 1, 250-256 (1992).

T.M. Peng, F.N. Hubele, \& G. Karady, An adaptive neural network approach to one-week ahead load forecasting, IEEE Transactions on Power Systems, 8: No. 3, 1195-1203 (1993). 